\title{
Ocorrência de protozoários gastrintestinais em primatas mantidos em cativeiro na região sul do Brasil
}

\author{
Occurrence of gastrointestinal protozoa in primates kept in captivity in the Southern \\ region of Brazil
}

\begin{abstract}
Aleksandro Schafer da Silva ${ }^{\mathrm{I}}$ Gabriela Pesamosca Coradini ${ }^{\mathrm{I}}$ Luciane Tourem Gressler $^{\mathrm{I}}$ João Fabio Soares ${ }^{\mathrm{I}}$ Valéria Maria Lara ${ }^{\mathrm{II}}$ Adriano Bonfim Carregaro ${ }^{\mathrm{III}}$ Silvia Gonzalez Monteiro ${ }^{\mathrm{II}}$
\end{abstract}

\section{- NOTA -}

RESUMO

Este trabalho visou avaliar o parasitismo gastrintestinal por protozoários em macacos mantidos em cativeiro na região Sul do Brasil. Foram analisadas amostras de fezes de 18 primatas de quatro espécies, Cebus apella, Macaca mulata, Callithrix jacchus e Callithrix penicillata pelo método de centrífugo flutuação com sulfato de zinco. Nos animais avaliados, foram verificados infecções simples e mistas pelos protozoários dos gêneros Cryptosporidium, Giardia, Cystoisospora e Balantidium. Analisando a água oferecida aos primatas foi detectada a presença elevada de oocistos de Cryptosporidium spp..

Palavras-chave: primatas, parasitismo, protozoários.

\section{ABSTRACT}

This research aimed at evaluating the gastrointestinal parasitism by protozoa in monkeys kept in captivity in the Southern region of Brazil. Fecal samples from 18 primates of the species Cebus apella, Macaca mulata, Callithrix jacchus and Callithrix penicillata were analyzed by the zinc sulphate centrifugal flotation method. Simple and mixed infections by protozoa of the genus Cryptosporidium, Giardia, Cystoisospora and Balantidium were observed. The water offered to the primates had elevated presence of oocysts of Cryptosporidium spp..

Key words: primates, parasitism, protozoa.

Os estudos sobre parasitos gastrintestinais em primatas mantidos em cativeiros são importantes para o manejo da população de macacos e para a manutenção da saúde das pessoas que trabalham com esses animais, pois muitos destes parasitos são potentes causadores de zoonoses. Também é importante verificar se esses animais se comportam como transmissores de parasitas para o meio ambiente por estarem infectados (BRACK, 1987).

As infecções e as parasitoses estão diretamente relacionadas com as condições de vida do hospedeiro e do ambiente onde estes vivem. Os parasitos gastrintestinais são mais prevalentes em locais onde não há boas condições de higiene, principalmente com a água e os alimentos consumidos (CARMO \& SALGADO, 2003).

Amostras de fezes de nove espécies de primatas (Alouatta seniculus, Aotus vociferans, Ateles bezlebuth chamek, Callicebus brunneus, Cebus albifrons, Cebus apella, Callithrix sp., Saguinus fuscicollis e Saimiri sciureus) oriundos de uma reserva nacional do Peru e do Brasil foram analisadas e foram detectados nestes macacos parasitismo pelos protozoários Blastocystis hominis, Chilomastix mesnili, Endolimax nana, Giardia intestinais, Entamoeba coli, Entamoeba histolytica e Iodamoeba buetschii, além de helmintos Ascaris sp., Strongyloides sp., Trichuris trichiura, Schistosoma mansoni e Prosthenochoris elegans (CARMO \& SALGADO, 2003; KIMBERLEY et al., 2004).

${ }^{\mathrm{I}}$ Curso de Medicina Veterinária, Universidade Federal de Santa Maria (UFSM), Santa Maria, RS, Brasil.

"Departamento de Microbiologia e Parasitologia, UFSM. Faixa de Camobi, Km 9, Campus Universitário, Prédio 20, Sala 4232, 97105-900, Santa Maria, RS, Brasil. E-mail: sgmonteiro@uol.com.br. *Autor para correspondência

IIIDepartamento de Pequenos Animais, UFSM, Santa Maria, RS, Brasil. 
Devido à carência de pesquisas envolvendo animais silvestres, este trabalho visou avaliar a ocorrência de protozoários gastrintestinais em macacos mantidos em cativeiro na região sul do Brasil. Foram analisadas amostras de fezes de 18 primatas de quatro espécies, sendo destes oito Cebus apella (macaco prego), quatro Macaca mulata (rhesus), quatro Callithrix jacchus (sagui-de-tufo-branco) e dois Callithrix penicillata (sagui-de-tufo-preto), oriundos de um zoológico e de um criatório conservacionista localizados nos municípios de Cachoeira do Sul e Santa Maria, no Rio Grande do Sul, Brasil. As amostras coletadas foram armazenadas sob refrigeração a $13^{\circ} \mathrm{C}$ até serem processadas em laboratório pelo método de centrífugo-flutuação com sulfato de zinco, pela técnica de Faust et al. (HOFFMANN, 1987) e pela técnica de coloração de Ziehl-Neelsen modificada por Padma (SIQUEIRA et al., 1984). A avaliação da carga parasitária foi baseada na classificação de PINTO et al. (1994), que qual considera-se infecção leve: 1-100 cistos ou oocistos, moderada: 101-300 cistos ou oocistos e elevada: mais de 301 cistos ou oocistos por lâmina. Os oocistos de coccídeos foram esporulados para identificação do gênero por meio da adição de dicromato de potássio a $\%$ nas fezes (HOFFMANN, 1987). Com a finalidade de identificar a fonte de infecção para os primatas, foram coletadas amostras de água do reservatório dos criatórios, as quais foram analisadas para pesquisa de protozoários pelo método de Yanko modificado (COELHO et al., 2002).

Nas amostras analisadas pela técnica de Faust e cols, observou-se parasitismo por Cryptosporidium spp. em seis $\boldsymbol{C}$. apella e em dois macacos de cada espécie, $\boldsymbol{M}$. mulata e $\boldsymbol{C}$. penicillata. Oocistos de Cystoisospora spp. foram encontrados nas fezes de quatro $\boldsymbol{C}$. apella, quatro $\boldsymbol{C}$. jacchus e dois $\boldsymbol{C}$. penicillata. A infecção por Giardia sp. foi identificada em quatro animais de duas espécies de primatas, isto é,
C. apella e C. jacchus. Em um dos macacos M. mulata foram observados oocistos de Balantidium sp. (Tabela 1). A técnica de coloração de Ziehl-Neelsen confirmou a presença de Cryptosporidium spp. nos primatas avaliados. Foi observado moderado grau de infecção para animais parasitados pelo gênero Cryptosporidium, já os demais protozoários encontravam-se apenas causando infecções leves nos macacos. Os animais analisados não apresentavam diarréia, um dos principais sinais clínicos das parasitoses por esses gêneros.

A infecção pelo gênero Cryptosporidium, verificada nos macacos deste estudo, já foi relatada em outros trabalhos realizados com primatas oriundos de florestas naturais do Sri Lanka, onde foi registrado o primeiro caso de parasitismo pelo protozoário nas espécies Macaca sinica, Presbytis entellus e Trachypithecus vetulus (EKANAYAKE et al., 2006). MURIUKI et al. (1997) e NIZEYI et al. (1999) avaliaram a prevalência de Cryptosporidium sp. em macacos (Cercopithecus aethiops) e gorilas (Gorilla gorilla beringei) e verificaram que $51,7 \%$ e $11 \%$, respectivamente, eram positivos a esse parasito. Além disso, esse coccídeo foi observado em várias espécies de macacos (Alouatta caraya, Ateles paniscus, Saimiri boliviensis, C. apella, Papio hamadryas e Pan troglodytes) no zoológico de Buenos Aires, Argentina (VENTURINI et al., 2006). Apesar do grande número de hospedeiros e da ampla distribuição geográfica deste protozoário, esta pesquisa trata-se do primeiro caso de parasitismo por Cryptosporidium sp. em primatas das espécies $\boldsymbol{M}$. mulata e $\boldsymbol{C}$. penicillata.

Conforme a literatura, os primatas podem ser parasitados por sete espécies do gênero Isospora (I. arctopitheci, I. belli, I. callimico, I. cebi, I. endocallimici, I. saimiriae e I. natalensis) (DUSZYNSKI et al., 1999). Neste estudo é descrito pela primeira vez o parasitismo por Cystoisospora sp. em $C$.

Tabela 1 - Protozoários gastrintestinais identificados em amostras de fezes de primatas mantidos em cativeiro no Rio Grande do Sul, Brasil.

\begin{tabular}{|c|c|c|c|c|}
\hline \multirow{3}{*}{ Gêneros identificados nas amostras de fezes } & \multicolumn{4}{|c|}{ 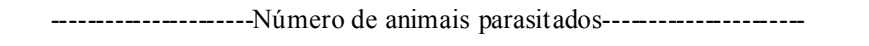 } \\
\hline & C. apella & M. mulatta & C. jacchus & C. penicillata \\
\hline & $\left(\mathrm{n}^{*}=8\right)$ & $(n=4)$ & $(n=4)$ & $(n=2)$ \\
\hline Cryptosporidium + Giardia + Cystoisospora & 04 & - & - & - \\
\hline Giardia + Cystoisospora & - & - & 04 & - \\
\hline Cryptosporidium + Cystoisospora & - & - & - & 02 \\
\hline Cryptosporidium & 02 & 02 & - & - \\
\hline Balantidium & - & 01 & - & - \\
\hline Negativos para protozoários & 02 & 01 & - & - \\
\hline
\end{tabular}

* Número de animais amostrados para cada espécie. 
apella, C. jacchus e C. penicillata, sendo que a infecção foi verificada em todos os animais do criatório de Santa Maria (Tabela 1). STUART et al. (1990) reportaram a ocorrência de Isospora sp. em bugio Alouatta palliata palliata e em Ateles geoffroyi oriundos de centros ecológicos na Costa Rica. Nas amostras de fezes analisadas neste estudo, foram observados raros oocistos desse protozoário com 17,4 x $20,7 \mu \mathrm{m}$ e esporocistos de $9,2 \times 11,1 \mu \mathrm{m}$. O tamanho dos oocistos foi semelhante entre os hospedeiros, embora não foi suficiente para identificar a espécie de Cystoisospora presente na infecção.

A giardiase é uma infecção comum em animais domésticos em todo o mundo, sendo atualmente identificada também em várias espécies de vertebrados selvagens, entre eles os primatas. O parasitismo por Giardia sp. é relatado em macacos das espécies Callithrix argentata, Cercopithecus erythrogaster, Macaca fuscata, M. mulatta e Alouatta pigra (MAJEWSKA \& KASPRZAK, 2000; SESTAK et al., 2003; ITAGAKI et al., 2005; VITAZKOVA \& WADE, 2006). A infecção por Giardia sp. em C.jacchus foi detectada pela primeira vez neste estudo, no entanto, o sagüi da espécie $\boldsymbol{C}$. argentata já foi reportado como um dos hospedeiros deste flagelado (MAJEWSKA \& KASPRZAK, 2000).

SESTAK et al. (2003) relataram a presença de oocistos de $\boldsymbol{B}$. coli nas fezes de M. mulatta, fato também verificado no presente trabalho. Há registros de prevalência de $84 \%$ desse protozoário em chimpanzés (P. troglodytes) e de $9 \%$ em macacos ( $\boldsymbol{M}$. fuscata) (NAKAUCHI et al., 1990; NAKAUCHI, 1999). Balantidium é considerado um comensal do trato intestinal dos suínos domésticos, agindo somente como invasor secundário. Além dos hospedeiros acima citados, há registros de ocorrências em macacos das espécies Cercopithecus neglectus, M. sinica, $P$. entellus e T. vetulus (KARERE \& MUNENE, 2002; EKANAYAKE et al., 2006).

Numerosos surtos de criptosporidiose devido à ingestão de água contaminada foram relatados ao redor do mundo (ROSE, 1997), sendo a proteção dos mananciais considerada como o melhor método de controle da infecção (SOLO-GABRIELE \& NEUMEISTER, 1996). Na água oferecida aos primatas do criadouro de Santa Maria, foi observada a presença elevada de oocistos de Cryptosporidium sp. e a amostra de água do zoológico de Cachoeira do Sul apresentava-se negativa para parasitas, todavia, nos dois ambientes avaliados, os macacos estavam infectados pelo coccídeo.

Este estudo relata pela primeira vez o parasitismo por protozoários dos gêneros
Cryptosporidium em M. mulata e C. penicillata, Giardia em C. jacchus, Cystoisospora em C. apella, C. jacchus e C. penicillata, possivelmente devido à carência de pesquisas na área, que é conseqüência principalmente da dificuldade de acesso à fauna e das restrições de órgãos ambientais.

\section{REFERÊNCIAS}

BRACK, M. Agents transmissible from simians to man. Springer-Verlag, Bedin. 1987, 454p. CARMO, A.M.; SALGADO, C.A. Ocorrência de parasitos intestinais em Callithrix sp. (Mammalia, Primates, Callithrichidae). Revista Brasileira de Zoociências, v.5, n.2, p.267-272, 2003.

COELHO, W.M. et al. Avaliação de metodologias para detecção de ovos de helmintos no lodo e determinação do percentual de recuperação. In:... XXVIII Congresso Interamericano de Ingeniería Sanitaria y Ambiental, Cancúm, México. Anais... 2002, v.CD. p.1-7.

DUSZYNSKI, D.W. et al. Coccidia (Apicomplexa: Eimeriidae) in the primates and the Scandentia. International Journal of Primatology, v.20, n.5, p.761-797, 1999.

EKANAYAKE, D.K. et al. Prevalence of Cryptosporidium and other enteric parasites among wild non-human primates in Polonnaruwa, Sri Lanka. The American Society of Tropical Medicine and Hygiene, v.74, n.2, p.322-329, 2006.

HOFFMAN, R, P. Diagnóstico de parasitismo veterinário. Porto Alegre: Sulina, 1987. 156p.

ITAGAKI, T. et al. Genotyping of Giardia intestinalis from domestic and wild animals in Japan using glutamete dehydrogenase gene sequencing. Veterinary Parasitology, v.133, n.4, p.283-287, 2005.

KARERE, G.M.; MUNENE, E. Some gastro-intestinal tract parasites in wild De Brazza's monkeys (Cercopithecus neglectus) in Kenya. Veterinary Parasitology, v.110, n.1-2, p.153-157, 2002 .

KIMBERLEY, A. et al. Survey of the gastrointestinal parasites of the primate community at Tambopata National Reserve, Peru. Journal of Zoology, v.264, 149-151, 2004.

MAJEWSKA, A.C.; KASPRZAK, W. New axenic isolates of Giardia from primates. Acta Parasitologica, v.45, n.1, p.5556,2000 .

MURIUKI, S.M. et al. The presence of Cryptosporidium oocysts in stools of clinically diarrheic and normal non-human primates in Kenya. Veterinary Parasitology, v.72, p.141-147, 1997.

NAKAUCHI, K. et al. Detection of Balantidium coli from evacuated feces in cynomolgus monkeys (Macaca fascicularis). Japanese Journal of Veterinary Science, v.52, n.6, p.1323-1324, 1990.

NAKAUCHI, K. The prevalence of Balantidium coli infection in fifty-six mammalian species. Journal of Veterinary Medicine Science, v.61, n.1, p.63-65, 1999. 
NIZEYI, J.B. et al. Cryptosporidium sp.and Giardia sp. infections in mountain gorillas (Gorilla gorilla beringei) of the Bwindi impenetrable national park, Uganda. Journal of Parasitology, v.85, p.1084-1088, 1999.

PINTO, R.M. et al. Helminth parasites of conventionally maintained laboratory mice. Memórias do Instituto Oswaldo Cruz, v.89, n.1, p.33-40, 1994.

ROSE, J.D. Environmental ecology of Cryptosporidium and public health implications. Annual Review of Public Health, v.18, p.135-161, 1997.

SESTAK, K. et al. Infectious agent and immune response characteristics of chronic Enterocolitis in aptive Rhesus Macaques. Infection and Immunity, v.71, n.7, p.4079-4086, 2003 .
SIQUEIRA, L.F.G. et al. Métodos tintoriais utilizados na identificação do Mycobacterium leprae: revisão histológica. Revista de Saúde Pública, v.18, n.3, p.246-258, 1984.

SOLO-GABRIELE, H.; NEUMEISTER, S. US outbreaks of cryptosporidiosis. Journal of the American Water Works Association, v.88, p.76-86, 1996.

STUART, M.D. et al. A coprological survey of parasites of wild mantled howling monkeys, Alouatta palliata palliata. Journal of wildlife diseases, v.26, n.4, p.547-549, 1990.

VENTURINI, L. et al. Cryptosporidium parvum en animales domésticos y em monos de un zoológico. Parasitologia Latinoamericana, v.61, p.90-93, 2006.

VITAZKOVA, S.K.; WADE, S.E. Parasites of free-ranging black howler monkeys (Alouatta pigra) from Belize and Mexico. American Journal of Primatology, v.68, n.11, p.1089-1097, 2006. 